\section{Hipoglicemia inducida por tumor fibroso solitario pulmonar. Síndrome de Doege-Potter}

\author{
JIMENA SOUTELO, SOFÍA MOLDES, AYELÉN MARTIN, \\ RUBÉN LUTFI, MARIELA LEAL REYNA
}

\section{Hypoglycemia induced by a solitary fibrous tumor of the lung or Doege-Potter syndrome. Report of one case}

We report the case of a 75-year-old man who was admitted to the hospital with symptoms of severe hypoglycemia. He had a history of solitary fibrous tumor diagnosed by Pathology after its complete surgical resection eight years before. The laboratory examination reported hypoglycemia with inhibited Insulin secretion. A computed tomography of the thorax revealed a large solid heterogeneous mass in the left hemithorax. Solitary fibrous tumor is a rare neoplasm. The association of solitary fibrous tumor and paraneoplastic hypoglycemia is known as Doege-Potter syndrome and occurs in less than 5\% of all solitary fibrous tumors.

(Rev Med Chile 2016; 144: 129-133)

Key words: Hypoglycemia, solitary fibrous tumor, non islet cell tumor hypoglycemia (NICTH).
Servicio de Endocrinología.

Complejo Médico Churruca Visca.

Buenos Aires. Argentina.

Conflicto de interés: Los autores no presentan conflicto de interés.

Recibido el 31 de julio de 2015, aceptado el 12 de noviembre de 2015.

Correspondencia a:

Dra. Jimena Soutelo Servicio de Endocrinología. Complejo Médico Churruca Visca. Uspallata 3400 (CP: 1437) Buenos Aires. Argentina. endochurruca@hotmail.com
L a hipoglicemia es una emergencia endocrina. Se define como una glicemia inferior de 55 $/ \mathrm{mg} / \mathrm{dl}$, en pacientes sin diabetes mellitus, asociada con signos y síntomas de hipoglicemia que revierten con glucosa oral o alimentos (Triada de Whipple). Su etiología puede deberse a hipersecreción de insulina o péptidos similares, incremento en la utilización periférica de glucosa o alteración de la secreción de hormonas contrarreguladoras.

En las neoplasias reconocidas como tumores de células no islotes (NICTH), la hipoglicemia es ocasionada por la producción del factor de crecimiento símil insulina tipo 2 (IGF-2) ${ }^{1}$.

Los NICTH constituyen un síndrome paraneoplásico poco frecuente, caracterizado por la presencia de un tumor sólido e hipoglicemia grave en ayunas causada por una vía independiente de la insulina. La incidencia y prevalencia de estos tumores son desconocidas pero se estima que son cuatro veces menos frecuentes que el insulinoma. En una revisión de 288 casos, $22 \%$ correspondieron a tumor fibroso solitario o mesoteliomas, $17 \%$ a carcinoma hepatocelular y $7 \%$ a hemangiopericitomas. Los carcinomas adrenales y carcinomas del tubo digestivo presentaron menor frecuencia. También se ha visto su asociación con leucemias, linfomas y feocromocitomas ${ }^{2}$.

El tumor fibroso solitario (TFS) tiene origen mesenquimático y su localización más frecuente es la pleura. Representa menos de $10 \%$ de los tumores pleurales ${ }^{3}$. Su incidencia es de 2,8 por 100.000 pacientes hospitalizados, Cardillo y col $^{4}$ realizaron una búsqueda sistemática y encontraron 1.760 casos publicados al 2012. Estos tumores son, en su mayoría, benignos, aunque $10-30 \%$ presentan características de malignidad ${ }^{3,5}$.

La asociación de hipoglicemia paraneoplásica y TFS, independientemente de su comportamiento biológico, se conoce como síndrome de 
Doege-Potter y ocurre en menos de $5 \%$ de todos los casos de TFS ${ }^{6-8}$.

Se presenta el caso debido a la baja incidencia del síndrome de Doege-Potter, la aparición alejada de la posible recidiva de la enfermedad, la importancia del manejo multidisciplinario de la patología y su tórpida evolución debido, no sólo al tumor primario, sino también a las comorbilidades del paciente.

\section{Caso clínico}

Paciente de sexo masculino de 75 años de edad, con escala ECOG 2 (Eastern Cooperative Oncology Group), que fue admitido en enero de 2015 en el Servicio de Clínica Médica por compromiso neurológico del sensorio, interpretado secundario a hipoglicemia (glicemia $38 \mathrm{mg} / \mathrm{dl}$ ). Presentaba antecedentes personales de ex tabaquismo, hipertensión arterial, infarto agudo de miocardio en 1995, cirugía de revascularización miocárdica en 2009 con realización de 4 bypass con miocardiopatía isquémico necrótica irrevascularizable e insuficiencia cardiaca con deterioro severo de la función sistólica (fracción de eyección 19\%). En el año 2006 se realizó resección completa de un tumor ubicado en base pulmonar izquierda compatible con TFS. La anatomía patológica informó proliferación fusocelular en patrón con crecimiento arremolinado y pseudocápsula fibrosa, leve a moderado pleomorfismo nuclear y 6 mitosis por 10 campos de $40 \mathrm{X}$, vimentina, CD34 y Bcl 2 positivos, Ki $6740 \%$ y S100 negativo ${ }^{3}$. En el año 2007, en la tomografía computada (TC) de control, no se evidenció lesión residual. Posteriormente, el paciente discontinuó sus controles por oncología, lo que impidió el correcto seguimiento y tratamiento de ser necesario. En el año 2014, al paciente se le diagnosticó cáncer de próstata con score de Gleason $8(4+4)$. Al realizar TC para estadificar dicho tumor, se evidenció una imagen de 110 x $70 \mathrm{~mm}$ en la base pulmonar izquierda (Figura 1) y aumento bilateral de glándulas adrenales mayor de glándula adrenal izquierda. La tomografía de emisión de positrones reveló una formación sólida heterogénea de 115 x 112 × $85 \mathrm{~mm}$ con áreas centrales hipermetabólicas, con valor máximo de captación estándar (SUVmax) 3,9 en región basal pulmonar izquierda y aumento difuso de la glándula adrenal izquierda, hipermetabólica con SUVmax 3. El análisis bioquímico permitió descartar patología adrenal hipo o hiperfuncionante. La resonancia magnética nuclear de glándulas adrenales reveló aumento de tamaño difuso de glándula adrenal izquierda, sin atenuación del parénquima en técnica de fase y fuera de fase, presentando restricción positiva con técnica de difusión y caída de los valores de coeficiente de difusión aparente. Compatible con lesión secundaria.

$\mathrm{Al}$ interrogatorio dirigido, el paciente refirió experimentar desde los últimos dos meses síntomas de hipoglicemia (diaforesis, palpitaciones y debilidad), alivio de los mismos con la ingesta de alimentos $\mathrm{y}$, en el último año, disnea clase funcional II-III (New York Heart Association). Presentó presión arterial: 100/70 $\mathrm{mmHg}$, frecuencia cardiaca: 80 latidos/min, frecuencia respiratoria: 22 respiraciones/min e índice de masa corporal: $23,9 \mathrm{~kg} / \mathrm{m}^{2}$, hipoventilación basal derecha y hasta campo medio izquierdo. En la exploración bioquímica efectuada a su ingreso, se constató cortisol basal: $16,6 \mu \mathrm{g} / \mathrm{ml}$ (VN: 6-19), sodio: $146 \mathrm{meq} / \mathrm{l}$ (VN 135-145), potasio: 3,4 meq/l (VN 3,5-5) con funciones tiroidea, renal y hepática conservadas. Durante la hospitalización el paciente evolucionó con episodios de hipoglicemia en ayunas, la exploración bioquímica de uno de estos episodios confirmó la presencia de hipoglicemia hipoinsulinémica: glicemia: $43 \mathrm{mg} / \mathrm{dl}$ [valor normal (VN)

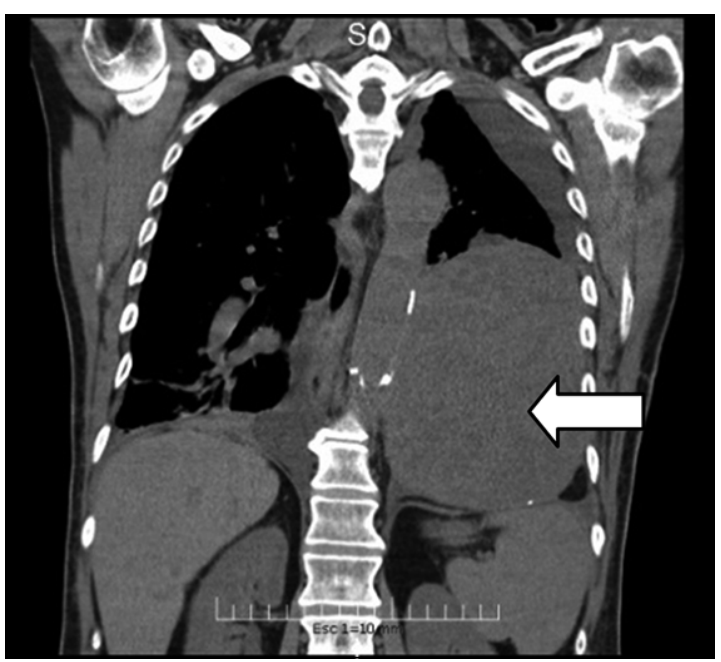

Figura 1. Tomografía computada de tórax sin contraste: Corte coronal que muestra masa de $110 \times 70 \mathrm{~mm}$ de contornos definidos en hemitórax izquierdo. 
70-110], insulina: $<1 \mu \mathrm{UI} / \mathrm{ml}$ (VN 4-18), péptido C: $0,12 \mathrm{ng} / \mathrm{ml}(\mathrm{VN} 0,8-4)$, IGF-1: $<25 \mathrm{ng} / \mathrm{ml}(\mathrm{VN}$ 55-175), IGFBP3: $0,8 \mathrm{ng} / \mathrm{ml}$ (VN 0,9-3,7), GH: $0,13 \mathrm{ng} / \mathrm{mL}(\mathrm{VN}<3)$. Se indicó tratamiento con solución dextrosada a $10 \%$ vía endovenosa y, debido a la persistencia de hipoglicemia, se inició meprednisona vía oral en dosis crecientes hasta alcanzar $20 \mathrm{mg}$ diarios, con lo cual normalizó los niveles de glicemias. En reunión clínico-quirúrgica multidisciplinario (Comité de Tumores), se decidió no tomar conducta quirúrgica ni iniciar radioterapia o quimioterapia debido a sus antecedentes cardiológicos. El paciente falleció como consecuencia de un infarto masivo de miocardio tres meses después de su egreso hospitalario.

\section{Discusión}

Los NICTH son tumores raros productores de IGF-2. Nadler y Wolfer describieron por primera vez un caso de hipoglicemia asociado con un carcinoma hepático, y los primeros en demostrar la presencia del factor de crecimiento insulino símil tipo 2 (IGF-2) en un tumor productor de hipoglicemia, fueron Daughaday y col. ${ }^{9}$. En el caso presentado y aunque no fue posible la medición de IGF-2 (en nuestro país no disponemos de los medios para su determinación), la exclusión de otras causas de hipoglicemia y la existencia de hipoglicemia hipoinsulinémica, niveles disminuidos de GH, IGF-1, IGFBP3, la rápida respuesta a los glucocorticoides y el antecedente de TFS, nos permitió establecer el diagnóstico presuntivo de $\mathrm{NICTH}^{1,2}$. Asimismo, destacamos que Hizuka y $\mathrm{col}^{10}$ demostraron 13 pacientes IGF-2 negativos en una serie de 44 pacientes con NICTH.

En 50\% de los NICTH se describe la asociación de hipoglicemia con hipopotasemia ${ }^{11}$. Ello resultaría de la acción del IGF-2 que, de igual modo a la insulina, favorecería el ingreso celular de potasio y disminuiría su concentración en plasma. En el caso presentado, la hipopotasemia leve observada, pudo estar enmascarada por el tratamiento con espironolactona.

El TFS pleural, en general, tiene un curso clínico benigno y excelente pronóstico. Afecta tanto a mujeres como varones y se presenta a cualquier edad, con una incidencia mayor en la sexta y séptima década de la vida. No obstante, el comportamiento biológico de estos tumores es variable y algunos muestran comportamiento maligno. Demostrar la estirpe celular mediante el análisis inmunohistoquímico es esencial para el diagnóstico adecuado ${ }^{12}$. En el caso presentado, la inmunomarcación vimentina, CD34 y Bcl-2 positivos simultáneamente con $\mathrm{S} 100$ negativo ${ }^{3}$, afirmaron la presencia de TFS. Además, el Ki-67 fue positivo al igual que en $5 \%$ de los casos reportados por Zhu y col. ${ }^{13}$.

En coincidencia con otros autores ${ }^{5,14} \mathrm{y}$ a diferencia de la mayoría de los TFS, el paciente presentó manifestaciones clínicas de hipoglicemia luego de 8 años de haber sido resecado el TFS, concurrentemente con probable recidiva de la enfermedad y con imágenes compatibles con extensión metastásica. Si bien existen controversias respecto de los factores pronósticos de estos tumores, en este caso, el riesgo de recurrencia era elevado si se considera: a) los marcadores pronósticos, establecidos por Franzen y col. ${ }^{15}$, que demostraron una asociación estadísticamente significativa entre el intervalo de supervivencia libre de enfermedad con el tamaño tumoral, el número de mitosis e índice de proliferación (Ki-67); b) Schmid y col ${ }^{12}$ constataron que la inmunomarcación con MIB-1 (Ki-67) podría tener, exclusivamente, un valor predictivo de recurrencia o sobrevida y c) el sistema de puntuación propuesto por Tapias y $\mathrm{col}^{16}$ que califica 6 características tumorales (origen, morfología, tamaño, hipercelularidad, presencia de necrosis o hemorragia y número de mitosis).

En cuanto al tratamiento, la resección quirúrgica del tumor productor de IGF-2 es el tratamiento de elección ${ }^{2}$. Sin embargo, la condición cardiológica de este paciente, el tamaño tumoral y la posible presencia de metástasis, imposibilitaron el tratamiento quirúrgico, embolización, quimioterapia (cardiotoxicidad) o radioterapia ${ }^{2}$.

El síndrome de Doege-Potter es poco frecuente, con una incidencia similar para ambos géneros. No existen diferencias en las características clínicas entre TFS malignos y benignos ni marcadores pronósticos específicos. Por lo tanto, resultan cruciales tanto el diagnóstico y tratamiento correcto, como el seguimiento ininterrumpido y multidisciplinario de estos tumores.

Asimismo, ante un paciente con hipoglicemia hipoinsulinémica, se debería pesquisar el antecedente o presencia de patología oncológica, con el fin de arribar al diagnóstico de NICHT (Figura 2). 


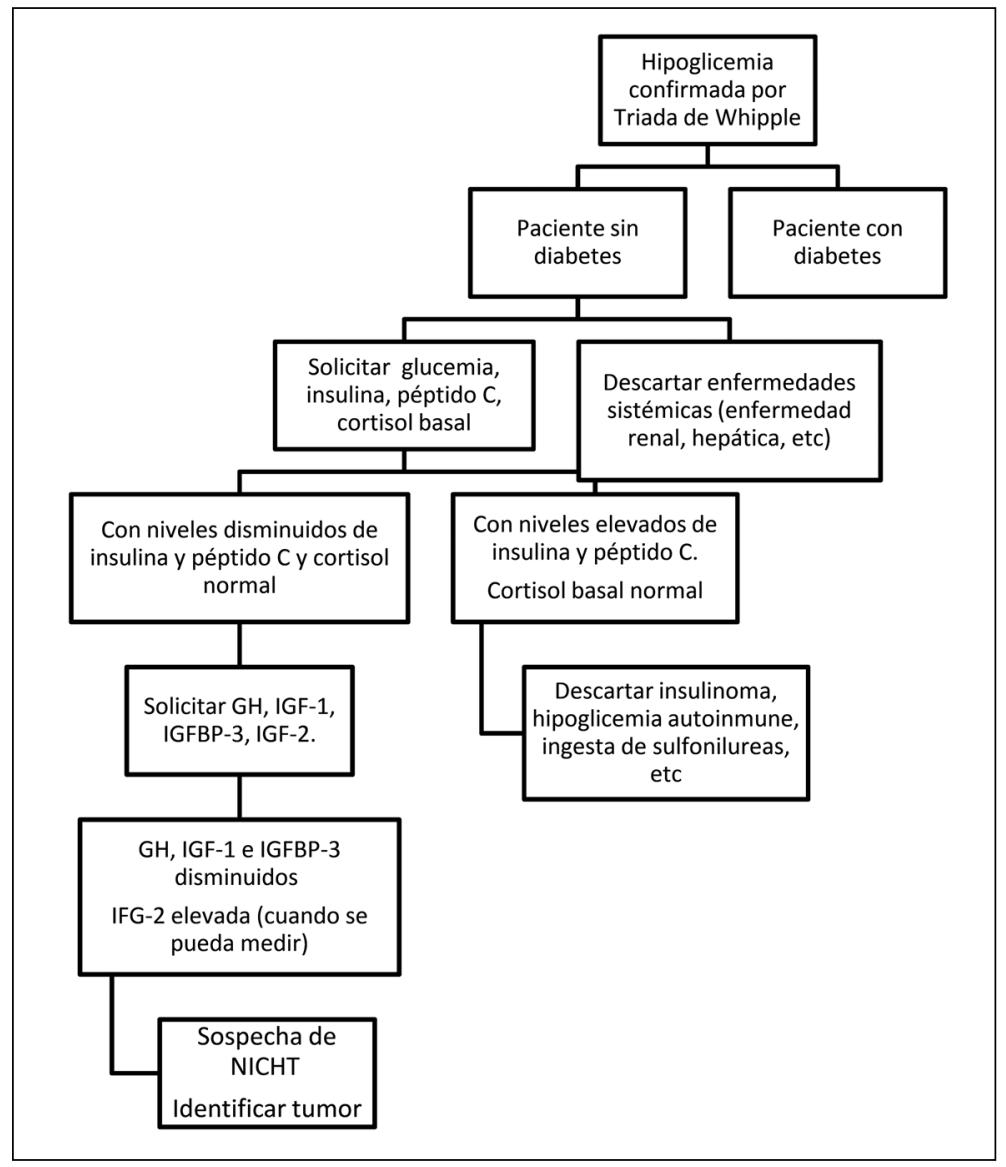

Figura 1. Algoritmo de hipoglicemia por tumor de células no islotes.

\section{Referencias}

1. Iglesias P, Diez JJ. A clinical update on tumor-induced Hypoglycemia. European Journal of Endocrinology 2014; 170: R147-57.

2. Bodnar TW, Acevedo MJ, Pietropaolo M. Management of Non-Islet-Cell Tumor Hypoglycemia. A Clinical Review. J Clin Endocrinol Metab 2014; 99: 713-22.

3. Sánchez-Mora N, Cebollero-Presmanes M, Monroy V, Carretero-Albiñana L, Herranz-Aladro M, Álvarez-Fernández E. Tumor fibroso solitario pleural: características clinicopatológicas de una serie de casos y revisión de la bibliografía. Arch Bronconeumol 2006; 42: 96-9.

4. Cardillo G, Lococo F, Carleo F, Martelli M. Solitary fibroustumors of the pleura. Curr Opin Pulm Med 2012; 18: 339-46.

5. Jeon HW, Kwon SS, Kim YD. Malignant solitary fibrous tumor of the pleura slowly growing over 17 years: case report. Journal of Cardiothoracic Surgery 2014, 9: 113.
Disponible en: http://www.cardiothoracicsurgery.org/ content/9/1/113.

6. Schutt RC, Gordon TA, Bhabhra R, Cathro HP, Cook SL, McCartney CR, et al. Doege-Potter syndrome presenting with hypoinsulinemic hypoglycemia in a patient with a malignant extrapleural solitary fibrous tumor: a case report. Journal of Medical Case Reports 2013; 7: 11. Disponible en: http://www.jmedicalcasereports.com/ content/7/1/11.

7. Jang JG, Chung JH, Hong KS, Ahn JH, Lee JH, Jo JH, et al. A Case of Solitary Fibrous Pleura Tumor. Associated with Severe Hypoglycemia: Doege-Potter Syndrome. Tuberc Respir Dis 2015; 78: 120-4.

8. Meng W, Zhu HH, Li H, Wang G, Wei D, Feng X. Solitary fibrous tumors of the pleura with Doege-Potter síndrome: a case report and three-decade review of the literatura. BMC research Notes 2014; 7: 515. http:// www.biomedcentral.com/1756-0500/7/515.

9. Daughaday WH, Emanuele MA, Brooks MH, Barbato 
AL, Kapadia M, Rotwein P. Synthesis and secretion of insulina-like growth factor II by a leiomyosarcoma with associated hypoglucemia. N Engl J Med 1988; 319: 143440.

10. Hizuka N, Fukuda I, Takano K, Okudo Y, Asakawa-Yasumoto K, Demura H. Serum insulina-like growth factor II in 44 patients with non-islet cell tumor hypoglucemia. Endocr J 1998; 45: S61-5.

11. Li Z, Wang J, Zhu Q, Li H, Chen Y, Chen L. Huge Solitary Fibrous Tumor of the Pleura with Hypoglycemia and Hypokalemia: A Case Report. Ann Thorac Cardiovasc Surg 2014; 20: 165-8.

12. Schmid S, Csanadi A, Kaifi JT, Kubler M, Haager B, Kayser G, et al. Prognostic factors in solitary fibrous tumors of the pleura. J Surgical Research 2015; 195: 580-7.

13. Zhu Y, Du K, Ye X, Song D, Long D. Solitary fibrous tumors of pleura and lung: report of twelve cases. J Thorac Dis 2013; 5 (3): 310-3.

14. Okimoto T, Horimasu Y, Hamaguchi S, Sutani A, Amano C, Harada Y, et al. Solitary Fibrous Tumor with Rapid Progression after 16 Years' Follow Up. Intern Med 2014; 53: 617-21.

15. Franzen D, Diebold M, Soltermann A, Schneiter D, Kestenholz P, Stahel R, et al. Determinants of outcome of solitary fibrous tumors of the pleura: an observational cohort study. BMC Pulmonary Medicine 2014; 14: 138.

16. Tapias LF, Mino-Kenudsaon M, Lee H, Wright C, Gaissert HA, Wain JC, et al. Risk factor analysis for the recurrence of resected solitary fibrous tumours of the pleura: a 33-year experience and proposal for a scoring system. Europen J of Cardio-Thoracic Surgery 2013; 44: 111-7. 